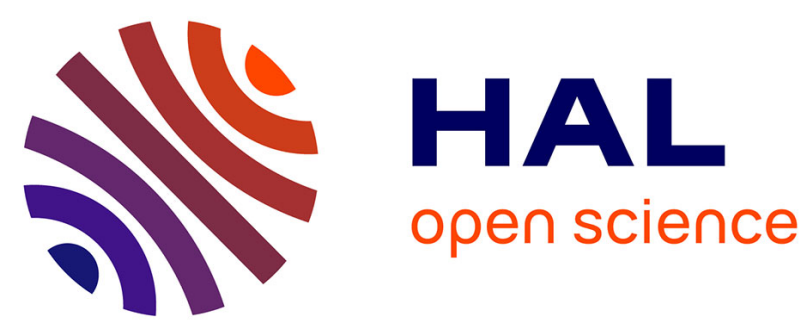

\title{
Equilibrium States of Generic Quantum Systems Subject to Periodic Driving
}

Achilleas Lazarides, Arnab Das, Roderich Moessner

\section{To cite this version:}

Achilleas Lazarides, Arnab Das, Roderich Moessner. Equilibrium States of Generic Quantum Systems Subject to Periodic Driving. Physical Review E : Statistical, Nonlinear, and Soft Matter Physics, 2014, 90, pp.012110. 10.1103/PhysRevE.90.012110 . hal-03019428

\section{HAL Id: hal-03019428 https://hal.science/hal-03019428}

Submitted on 23 Nov 2020

HAL is a multi-disciplinary open access archive for the deposit and dissemination of scientific research documents, whether they are published or not. The documents may come from teaching and research institutions in France or abroad, or from public or private research centers.
L'archive ouverte pluridisciplinaire $\mathbf{H A L}$, est destinée au dépôt et à la diffusion de documents scientifiques de niveau recherche, publiés ou non, émanant des établissements d'enseignement et de recherche français ou étrangers, des laboratoires publics ou privés. 


\title{
Equilibrium states of generic quantum systems subject to periodic driving
}

\author{
Achilleas Lazarides, ${ }^{1}$ Arnab Das, ${ }^{2}$ and Roderich Moessner ${ }^{1}$ \\ ${ }^{1}$ Max-Planck-Institut für Physik komplexer Systeme, 01187 Dresden, Germany \\ ${ }^{2}$ Theoretical Physics Department, Indian Association for the Cultivation of Science, Kolkata 700032, India
}

(Received 11 March 2014; revised manuscript received 27 June 2014; published 11 July 2014)

\begin{abstract}
When a closed quantum system is driven periodically with period $T$, it approaches a periodic state synchronized with the drive in which any local observable measured stroboscopically approaches a steady value. For integrable systems, the resulting behavior is captured by a periodic version of a generalized Gibbs ensemble. By contrast, here we show that for generic nonintegrable interacting systems, local observables become independent of the initial state entirely. Essentially, this happens because Floquet eigenstates of the driven system at quasienergy $\omega_{\alpha}$ consist of a mixture of the exponentially many eigenstates of the undriven Hamiltonian, which are thus drawn from the entire extensive undriven spectrum. This is a form of equilibration which depends only on the Hilbert space of the undriven system and not on any details of its Hamiltonian.
\end{abstract}

DOI: 10.1103/PhysRevE.90.012110

PACS number(s): 05.30.Ch, 37.10.Jk, 67.85.-d

\section{INTRODUCTION}

There has been intense recent interest in equilibration and thermalization of closed quantum systems. If large enough, such systems approach a steady state well described by the usual constructs of statistical mechanics. The effort to understand the mechanisms by which unitary quantum evolution leads to time-independent states, which can be characterized by fixing a reasonably small number of observables, as it must if statistical mechanics is to apply, has been one of the most fruitful in nonequilibrium quantum dynamics [1-7].

At the same time, much experimental and theoretical effort has been devoted to periodically driven systems [8]. The formal framework has been mostly set up by Shirley [9] and Sambe [10] and has been successfully applied in various fields, such as NMR [11,12], nonlinear optics [13], and others [14-16]. Closer to the subject of this work, it has recently been shown that isolated many-body periodically driven systems eventually synchronize into a periodic steady state with the driving $[17,18]$, in analogy with closed, nondriven systems approaching a stationary equilibrium state.

In a recent article [18], we have taken a first step towards characterizing the long-time synchronized state, by obtaining a description of the long-time steady state of an integrable system analogous to the generalized Gibbs ensemble (GGE) [19] for undriven systems, finding that memory of the relevant conserved quantities persists for all time.

Here we study the generic situation of a nonintegrable periodically driven model. Remarkably, we find that the longtime behavior is stationary and independent of both the initial condition and details of the undriven Hamiltonian beyond its Hilbert space.

We give a physical mechanism explaining this result: the expectation values of observables in any eigenstate are the same for all eigenstates. This is caused by the width of the quasienergy spectrum being finite, whereas that of the energy spectrum of the undriven Hamiltonian is extensive. This leads to a perturbation theory in the driving having vanishing radius of convergence, instead immediately mixing any initial state with a finite fraction of the states of the entire spectrum in the thermodynamic limit.
The importance of this feature of the quasienergy spectrum appears to have been appreciated first by Hone, Ketzmerick, and Kohn in the context of continuum single-particle problems $[20,21]$. Our result is also in keeping with a very recent preprint of D'Alessio and Rigol, who argued that closed driven quantum systems tend to a circular ensemble of random matrix theory, which they interpret as an infinite temperature state [22].

The remainder of this paper is organized as follows. We first define the problem and introduce notation, before deriving our central result of the existence of a steady state which is independent of all of time, driving, and the undriven Hamiltonian, depending only on the Hilbert space. We then analyze a particular model Hamiltonian numerically in order to demonstrate the correctness of the central ingredients of our analysis. Finally we discuss the difference with integrable systems, and conclude with an outlook.

\section{SETUP}

We consider a periodically driven system described by the Hamiltonian

$$
H(t)=H_{S}+u h_{D}(t)
$$

with $H_{S}$ time-independent and nonintegrable and $h_{D}(t+T)=$ $h_{D}(t)$ the periodically driven part, with $u$ a driving amplitude with units of energy.

We shall take $H_{S}$ to satisfy the eigenstate thermalization hypothesis $(\mathrm{ETH})$ : eigenstates that are close in energy look "similar" [23]; this notion has been made more concrete recently $[1-3,24]$. Following these, we say that the ETH is satisfied for a certain operator $\hat{O}$ if the eigenstate expectation values (EEVs) of $\hat{O}$, defined as $\left\langle\varepsilon_{\alpha}|\hat{O}| \varepsilon_{\alpha}\right\rangle$ with $|\varepsilon\rangle$ an energy eigenstate of energy $\varepsilon$, form a smooth function of the eigenstate energy $\varepsilon$ in the thermodynamic limit. Thus the mean energy of a macroscopic system fixes the expectation value of $\hat{O}$, and a small variation in the energy results in a small variation in the expectation value. Had this not been the case, arbitrarily small (microscopic) changes in energy would result in vastly different expectation values of the operator on macroscopic scales. The ETH has been confirmed to occur in a number of systems $[3,24,25]$. 
Adding a periodically driven part, $u h_{D}(t)$, to $H_{S}$ necessitates a change of viewpoint: instead of eigenstates and eigenenergies, one considers Floquet states and quasienergies. These are obtained from the eigenfunctions and eigenvalues of the operator

$$
U(\epsilon, \epsilon+T)=\mathcal{T} \exp \left[-i \int_{\epsilon}^{\epsilon+T} d t H(t)\right]
$$

from which one may define an effective Hamiltonian via $\exp \left[-i H_{\text {eff }}(\epsilon) T\right]=U(\epsilon, \epsilon+T)$. Its eigenvectors $|\alpha(\epsilon)\rangle$ satisfy $|\alpha(\epsilon)\rangle=|\alpha(\epsilon+T)\rangle$, while its eigenvalues have the form $\exp \left(-i \omega_{\alpha} T\right)$, with quasienergies $\omega_{\alpha}$ independent of $\epsilon$. The Floquet states, forming a complete set for equal-time properties, are defined as $\left|u_{\alpha}(t)\right\rangle=\exp \left(-i \omega_{\alpha} t\right)|\alpha(t)\rangle$.

Note that, as behooves a periodic system, the quasienergies necessarily lie in a "Brillouin zone" (BZ) of finite, nonextensive width $\omega=2 \pi / T$; this feature will play a crucial role in our analysis.

Starting from an initial state, after a transient period synchronization with the driving is achieved $[17,18]$ in the following sense: Take an initial density matrix $\hat{\rho}(0)=$ $\sum_{\alpha, \beta} \rho_{\alpha, \beta}|\alpha(0)\rangle\langle\beta(0)|$; at long times, the system behaves indistinguishably $[6,7]$ from one described by

$$
\hat{\rho}_{D E}(t)=\sum_{\alpha} \rho_{\alpha, \alpha}|\alpha(t)\rangle\langle\alpha(t)|,
$$

which is evidently periodic in time. The expectation value of an operator $\hat{O}$ in this state is

$$
\mathcal{O}(t)=\sum_{\alpha} \rho_{\alpha, \alpha} O_{\alpha, \alpha}(t)
$$

with the eigenstate expectation values (EEVs) $O_{\alpha, \alpha}(t)=$ $\langle\alpha(t)|\hat{O}| \alpha(t)\rangle$. This is analogous to the so-called diagonal ensemble (DE) for nondriven systems; in principle, this depends on the initial state through the quantities $\rho_{\alpha, \alpha}$.

\section{EIGENSTATE MIXING}

We begin by discussing the eigenvectors and quasinergies of $H_{\text {eff }}(\epsilon)$. For vanishing $u$ (alternatively, for stroboscopic observations of the system in the absence of any driving), the eigenstates $|\alpha(\epsilon)\rangle$ are time independent and identical to those of $H_{S}$. The corresponding quasienergies are thus $\omega_{\alpha}=\bmod \left(\varepsilon_{\alpha}, \omega\right)$. This implies that, even if the (nondriven) system $H_{S}$ satisfies the ETH for some observable, labeling the eigenstates by $\omega_{\alpha}$ instead of $\varepsilon_{\alpha}$ will in general destroy this property: the EEVs will not be a smooth function of the quasienergy, since now eigenstates whose energy differs by an integer number of "reciprocal lattice vectors" $\omega=h / T$ (with $h$ Planck's constant) have the same quasienergy. By continuity, one might expect this to remain true for "small" $u$. However, this expectation turns out to be wrong in the thermodynamic limit.

One may see this from the results of Refs. [20,21], from which the following picture emerges: Suppose we fix a $u$ and calculate the states $|\alpha(\epsilon)\rangle$. If $u \rightarrow u+\delta u$, one might hope to use perturbation theory to obtain the new states. However, the quantity compared to which $\delta u$ needs to be small is the quasienergy level spacing. As the dimension of Hilbert $D_{H}$ increases exponentially with system size, and the width of the quasienergy BZ is independent of it, the level spacing is exponentially small, and hence so is the radius of convergence of such a perturbation theory; one cannot expect adiabatic evolution. In particular, the basis states at arbitrarily small $u$ are not perturbatively related to the undriven ones in the thermodynamic limit; and an arbitrarily small change in $u$ mixes the $|\alpha(\epsilon)\rangle$ among themselves. This mixing implies that each $|\alpha(\epsilon)\rangle$ contains contributions from a finite fraction of the undriven states, uniformly spread over the entire spectrum. We have confirmed this explicitly by calculating the average participation ratio of the eigenstates of $H_{\text {eff }}(\epsilon)$ in the basis of the eigenstates of $H_{S}$ (see Appendix A).

Given this strong mixing across the entire spectrum of $H_{S}$, it is thus natural to expect that expectation values with respect to the $|\alpha(\epsilon)\rangle$ effectively average essentially uniformly over those with respect to the eigenstates of $H_{S}$, as captured qualitatively by the following rough argument. Let us expand the former in terms of the latter, $|\alpha(\epsilon)\rangle=$ $\sum_{n}|n\rangle\langle n \mid \alpha(\epsilon)\rangle$, and replace $\langle\alpha(\epsilon) \mid m\rangle \approx 1 / \sqrt{D_{H}} \exp \left(i \phi_{m}(\epsilon)\right)$ with the phases $\phi_{m}(\epsilon)$ uncorrelated between different $m$. Note that for this replacement to be reasonable, provided a smooth dependence of $\langle m|\hat{O}| m\rangle$ on $\varepsilon_{m}$, it is not necessary that all overlaps $\langle\alpha(\epsilon) \mid m\rangle$ are finite; rather, a sufficient condition is that the ones that are finite are uniformly and densely spread throughout the band of $H_{S}$, which we numerically observe (see Appendix A). Then, $\langle\alpha(\epsilon)|\hat{O}| \alpha(\epsilon)\rangle \approx$ $D_{H}^{-1} \sum_{m, n} \exp \left\{i\left[\phi_{n}(\epsilon)-\phi_{m}(\epsilon)\right]\right\}\langle m|\hat{O}| n\rangle$. Finally, given that (a) the phases are uncorrelated and (b) $\langle m|\hat{O}| n\rangle$ decreases rapidly with increasing $\left|\varepsilon_{m}-\varepsilon_{n}\right|$, as occurs in nonintegrable systems, and assuming that none of the $\langle m|\hat{O}| n\rangle$ grows with $D_{H}$ (in other words, that the observable is not localized in the basis of the $|m\rangle)$ we find $\langle\alpha(\epsilon)|\hat{O}| \alpha(\epsilon)\rangle \approx$ $\frac{1}{D_{H}} \sum_{m}\langle m|\hat{O}| m\rangle=D_{H}^{-1} \operatorname{tr}(\hat{\mathrm{O}})$, independent of both $\alpha$ and $\epsilon$.

This implies that the long-time steady state of the observable is not just periodic, but in fact even independent of time. In addition, since Eq. (4) becomes

$$
\mathcal{O}(t)=O(t) \sum_{\alpha} \rho_{\alpha, \alpha}=D_{H}^{-1} \operatorname{tr}(\hat{\mathrm{O}}),
$$

so that the long-time state is even completely independent of the initial condition (encoded in $\rho_{\alpha, \alpha}$ ).

We now turn to the numerical confirmation of the ingredients of our above analysis for a particular instance of a driven model system.

\section{EIGENSTATE EXPECTATION VALUES}

We consider hardcore bosons $\left(b_{i}^{2}=0\right)$ with

$$
\begin{aligned}
H(t)= & -\frac{1}{2} \sum_{i} b_{i}^{\dagger} b_{i+1}+\text { H.c. }+V_{1} \sum_{i} n_{i} n_{i+1} \\
& +V_{2} \sum_{i} n_{i} n_{i+2}+u \sum_{i} V_{i}(t) n_{i}
\end{aligned}
$$

featuring a potential $V_{i}(t)=\widetilde{u}(t)(-1)^{i}$ with $\widetilde{u}(t)=+1$ for $0<t<T / 2$ and $\widetilde{u}(t)=-1$ for $T / 2 \leqslant t \leqslant T$ (see Appendix $C$ for another example). Throughout, $J=V_{1}=V_{2}=1$.

For this system [26], we calculate the EEVs of the (arbitrarily chosen) local density operator $b_{8}^{\dagger} b_{8}$, plot them, and 


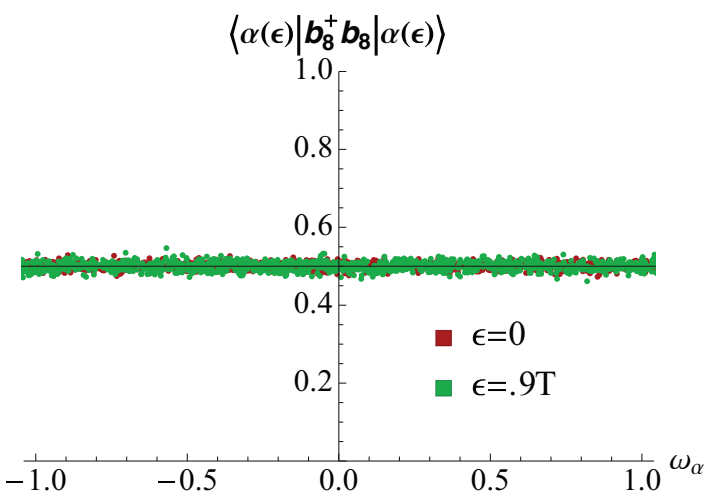

FIG. 1. (Color online) Example of the EEV dependence on the quasienergy $\omega_{f}$ for $u / \hbar \omega=1$ and system size and particle number $L=14, N=7$ for a Hilbert space dimension $D_{H}=3432$, with parameters $u=V_{1}=V_{2}=J$ and driving frequency $\hbar \omega=h / T=$ $J / 4$. Points indicate expectation value of the density at site $i=8$ in an eigenstate $|\alpha(\epsilon)\rangle$ of $H_{\text {eff }}(\epsilon)$ versus the state's quasienergy $\omega_{\alpha}$ at two different times. The black line indicates $\operatorname{tr}\left(b_{8}^{\dagger} b_{8}\right)=N / L=0.5$.

compare to the mean EEV in a window centered at the current EEV; an example is shown in Fig. 1 [27]. As expected, the EEVs show no dependence on quasienergy. Indeed, this result seems to be natural absent a preferred choice of origin of the quasienergy BZ.

We next study the approach to the thermodynamic limit. To do this, we define a root mean square deviation of the EEVs. Taking an average over a window of $w+1$ states, $\bar{O}_{\alpha}=$ $\frac{1}{w} \sum_{\beta} O_{\beta, \beta}$ with $\beta$ running from $\alpha-w / 2$ to $\alpha+w / 2$, the root mean square deviation is $V^{2}=\frac{1}{D_{H}} \sum_{\alpha}^{D_{H}}\left(O_{\alpha \alpha}-\bar{O}_{\alpha}\right)^{2}$. We are interested in whether and how $V$ vanishes with increasing $D_{H}$. By numerically fitting its behavior, we find that $V=c D_{H}^{\alpha}$ (see Appendix B for an example fit); the exponent $\alpha$ for a number of different $D_{H}$ (which we vary by varying the system size $L$, and the number of particles $N$ ) and two observables, the density at site $i=8$ and the operator $b_{3}^{\dagger} b_{4}$, is shown in Fig. 2.

From this, $\alpha$ appears to be independent of $u$ and approximately equal to $-1 / 2$; the upwards shift for small $u$ is a finitesize effect, as $u$ becomes too small given the level spacing of

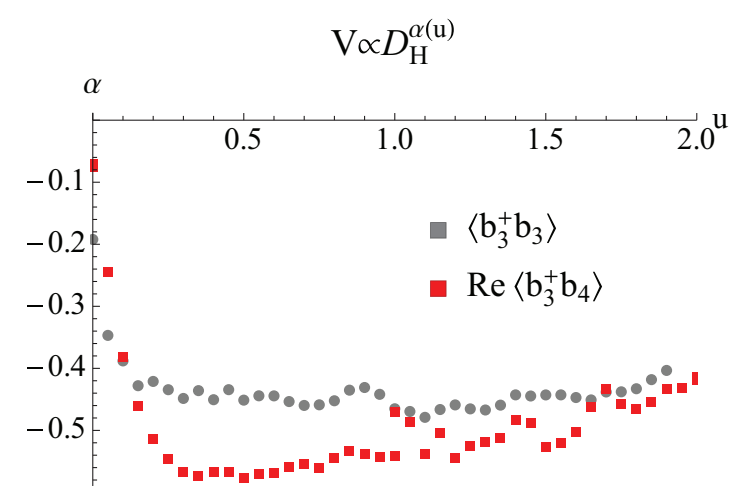

FIG. 2. (Color online) Fitted exponent $\alpha$ vs driving amplitude $u$, extracted for the observable $b_{3}^{\dagger} b_{4}$ as well as the density at site $i=3$ for the Hamiltonian of Eq. (5) for driving period $\omega=2 \pi / T=1$. The upturn at small $u$ is a finite-size effect. the system sizes we have access to. We therefore conclude that for a large enough system, the EEVs $O_{\alpha, \alpha}$ become independent of $\alpha$ in the thermodynamic limit as expected.

\section{DYNAMICS}

Having confirmed that the EEVs are all equal, we now confirm that this does indeed lead to independence of the final state from the initial state. To this end we explicitly calculate the dynamics starting from different initial states and check whether the final state is the same. We follow the following protocol. Diagonalizing the Hamiltonian of Eq. (5) with $J=V_{1}=V_{2}=1$ and a diagonal potential $V_{i}=i^{2}$, we select three states: the ground state, the eigenstate $1 / 4$ of the way up from the ground state, and the state in the middle of the band. We then switch off the diagonal potential and, for each state, calculate the time evolution under periodic driving with, again, $J=V_{1}=V_{2}=1, u=5 J$ and $V_{i}(t)=u(t)(-1)^{i}$ with $u(t)=+u$ for $0<t<T / 2$ and $u(t)=-u$ for $T / 2 \leqslant t \leqslant T$, as in the preceding discussion. At the beginning of each period, we calculate the instantaneous expectation of the Hamiltonian of Eq. (5). The results are displayed in Fig. 3: for two different system sizes, the expectation value of the instantaneous energy evolves to the same value in all three states, as do the expectation values of the operator $b_{3}^{\dagger} b_{4}$.

\section{DISCUSSION}

Taking a step back, we recognize two things happening here. First, at long times the system approaches a steady state [Eq. (4)], which is in principle periodic in time. Second, and more surprisingly, the EEVs are independent of the quasienergy, which leads to the synchronized state being independent of the initial condition. It is rather a property of the basic degrees of freedom of the system only, such as their locality and the Hilbert space they span, being essentially independent of any further "details" of the Hamiltonian. The system therefore loses all memory of the initial state, unlike the situation in either nondriven systems undergoing a quench or integrable driven systems [18].

The necessary ingredient is the absence of an adiabatic limit as $u$ is varied for large enough systems [20,21]. This causes an arbitrarily small change in $u$ to mix all eigenstates together; applying this to $u$ close to the undriven limit $u=0$, we see that the information contained in the dependence of the EEVs on energy, which determines the macroscopic properties of the system as a function of its energy, is completely scrambled. The final state mixes together macroscopic properties of undriven states at all energies and ends up completely featureless as a result.

By contrast, for a nondriven system, a finite-strength perturbation only couples unperturbed eigenstates within a finite fraction of the energy band. As a consequence, the EEVs of any operator in the perturbed basis are sensitive only to the unperturbed EEVs from nearby energies. This results in the perturbed EEVs remaining energy dependent and, in general, continuous.

The fact that this does not occur for integrable driven systems, where a periodic generalized Gibbs ensemble is found instead [18], seems at odds with the generality of the above 

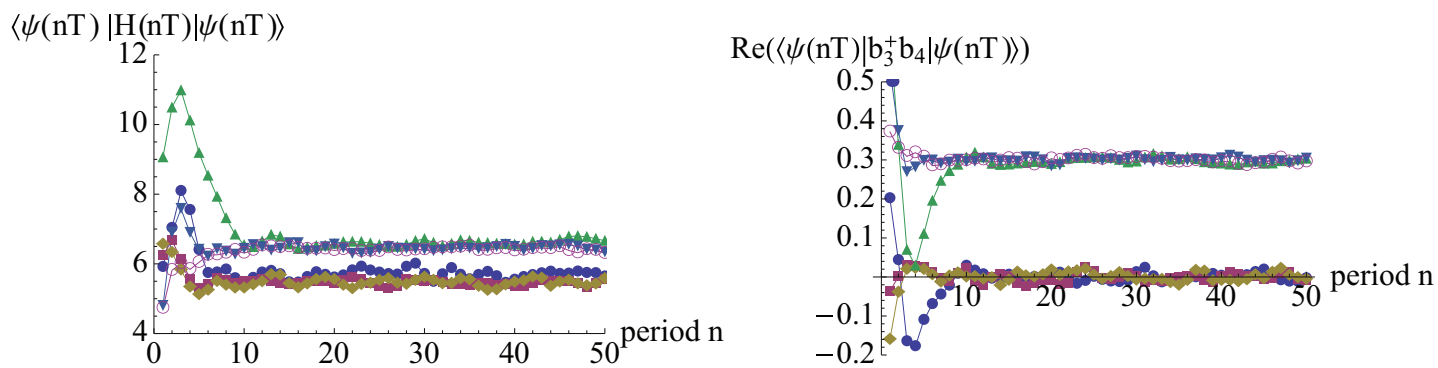

FIG. 3. (Color online) Left: Dynamical evolution of instantaneous value of the energy at the beginning of each period. The blue, gold red, and gold data points (three lower lines) correspond to different states, selected from different parts of the band of a Hamiltonian (which is different from the Hamiltonian used during the driving) and for for $L=12, N=6$ so $D_{H}=924$. The three top lines show time evolution of the same states, but for for $L=14, N=7$ so $D_{H}=3003$. In both cases, $u / \hbar \omega=5$. Right: Similar results are obtained for other observables, such as $b_{3}^{\dagger} b_{4}$. The bottom three lines are for $L=12, N=6$ while the top three lines are for $L=14, N=7$, and are offset vertically for clarity (in reality, they also oscillate about 0 ).

arguments. However, note that the extensive number (proportional to system size $L$ ) of conserved quantities exponentially reduces the number of states which get mixed together, as fixing $L$ quantities independently leads to a Hamiltonian matrix block diagonal with exponentially many uncoupled blocks. In those cases where the driving does not couple the different blocks (as it does not for systems mappable to free fermions via a Jordan-Wigner transformation, for instance) the scrambling of the eigenstates described above happens only inside each (small) block of size polynomial in $L$. This is not sufficient to randomize the eigenvectors, so that $O_{\alpha \alpha}(t)$ is neither $t$ - nor $\alpha$-independent and cannot be pulled out of the sum on the right-hand side of Eq. (4). Therefore, the long-time state is sensitive to the (initial state dependent) form of $\rho_{\alpha, \alpha}$.

Finally, we note that our results might be inapplicable to systems with infinite local Hilbert spaces, such as nonhardcore bosonic or continuum systems. This follows from the fact that the diagonal ensemble result for the long-time expectation value of the instantaneous energy density $\operatorname{tr}(H(n T)) / L$ diverges.

Several avenues for future work immediately suggest themselves. First, it will be interesting to study the approach to the steady state as a function of time and system size. Second, it will be interesting to study and classify the effect of driving for systems not obeying ETH in the undriven limit. Finally,

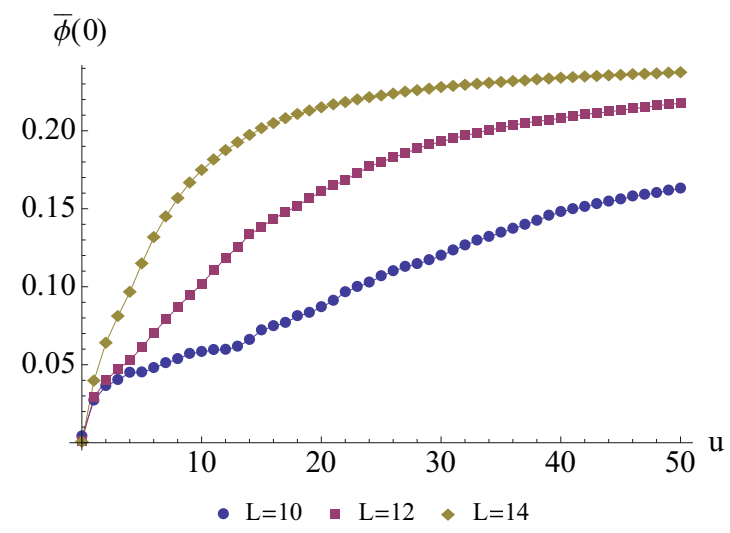
sizes. given our analysis was phrased largely perturbatively in $u$, it is not entirely clear what happens when $u$ becomes arbitrarily large.

\section{ACKNOWLEDGMENTS}

A.L. thanks W. Beugeling, A. Eckardt, and O. Tieleman for insightful observations throughout the course of this work. A.D. thanks A. Sen for discussions and MPI-PKS for hospitality during the course of this work.

\section{APPENDIX A: PARTICIPATION RATIOS}

Define the participation ratio (PR) $\phi_{\alpha}(\epsilon)=$ $\left[D_{H} \sum_{n=1}^{D_{H}}|\langle n \mid \alpha(\epsilon)\rangle|^{4}\right]^{-1}$ and its average $\bar{\phi}(\epsilon)=$ $D_{H}^{-1} \sum_{\alpha=1}^{D_{H}} \phi_{\alpha}(\epsilon)$; the quantity $\phi_{\alpha}(\epsilon)$ is $1 / D_{H}$ if a single $|n\rangle$ has finite overlap with $|\alpha(\epsilon)\rangle$ and becomes 1 if every single $|n\rangle$ participates equally in $|\alpha(\epsilon)\rangle$. It therefore roughly measures the fraction of the eigenstates of $H_{S}$ mixed into $|\alpha(\epsilon)\rangle$. For convenience, we also define

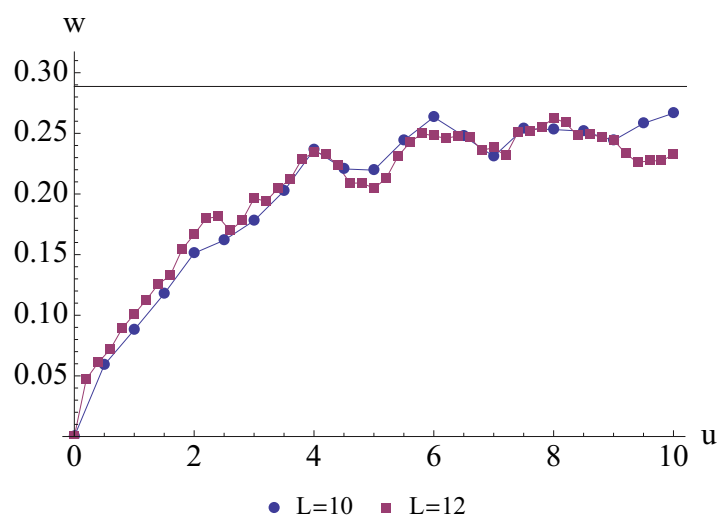

FIG. 5. (Color online) Width of bandwidth of $H_{S}$ participating in each eigenstate of $H_{\text {eff }}(0)$, averaged over all its eigenstates for the superlattice Hamiltonian described in the text. The solid line indicates the result for the limit in which all states participate equally. 


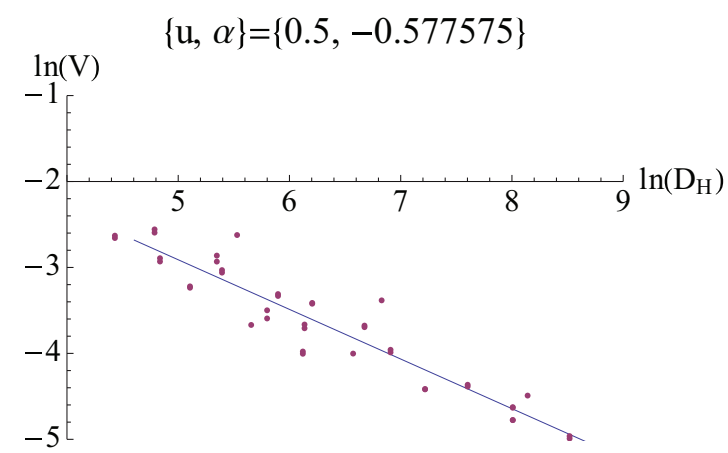

FIG. 6. (Color online) Best fit and data points for $u=0.5$. This figure corresponds to a single data point in Fig. 2. It is obtained by varying $L$, the system size, and $N$, the number of particles.

$\bar{m}=\frac{1}{\sum_{m} f_{m}} \sum_{m} m f_{m}$ with $f_{m}=|\langle\alpha(\epsilon) \mid m\rangle|$, the mean position of the participating eigenstates, and a "radius of gyration," $w^{2}=\frac{1}{\sum_{m} f_{m}} \sum_{m}(m-\bar{m})^{2} f_{m}$. This roughly indicates how much of the bandwidth of $H_{S}$ is involved in each eigenstate of $H_{\text {eff }}(0)$. Together, these quantities allow us to show two things: first, that a finite fraction of eigenstates of $H_{S}$ participate in each $|\alpha(0)\rangle$, and, second, that the entire bandwidth of $H_{S}$ participates in each $|\alpha(0)\rangle$.

Figure 4 shows the average participation ratios $\phi(0)$ for a number of system sizes as a function of $u$. Evidently the fraction of undriven eigenstates involved in each $|\alpha(0)\rangle$ is finite. Figure 5 then shows that the participating states are not concentrated in some region of the spectrum of $H_{S}$ but rather occupy the entire bandwidth (compare the black line which indicates the result for a uniform distribution throughout the band).

\section{APPENDIX B: EXAMPLE RESULTS FOR EEV VARIANCE VERSUS SYSTEM SIZE}

Figure 6 shows how the exponent $\alpha$ for the scaling of the EEV variance $V$ with Hilbert space dimension $D_{H}$ is extracted. The results shown in Fig. 2 of the main text are obtained by repeating this for different values of $u$.

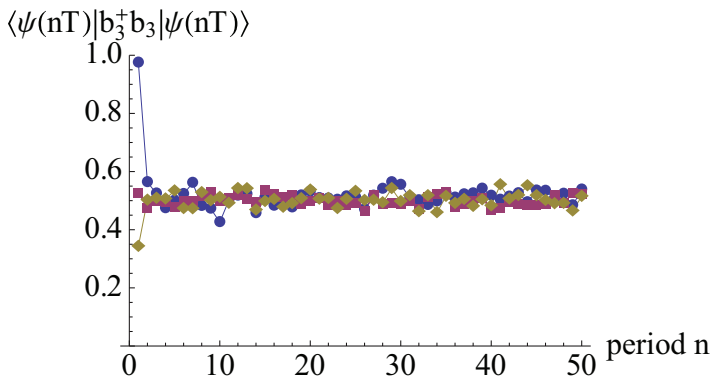

FIG. 7. (Color online) Stroboscopic observation of the density at site $i=3$ for a breathing trap as a function of period. The initial states are prepared as described in the main text, and the observations are made at the beginning of the period, $\epsilon=0$.

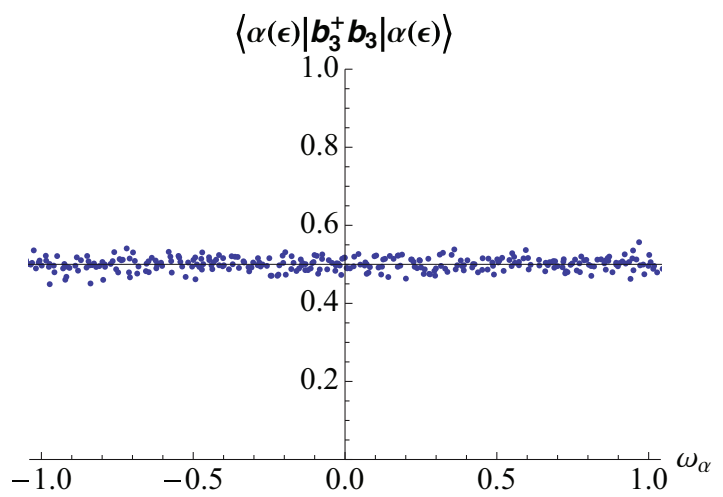

FIG. 8. (Color online) Eigenstate expectation values for the density at site $i=3$ for a breathing trap for $\epsilon=0$.

\section{APPENDIX C: BREATHING-TRAP POTENTIAL}

Here we show plots of EEVs, dynamics, and the final state for a Hamiltonian of the same form as in the main text [Eq. (5)] but with a time-dependent potential

$$
V_{i}(t)=\left[(i-L / 2) / \ell_{h o}(t)\right]^{2}
$$

with $\ell_{h o}(t)=\ell_{0}+\tilde{u}(t) \delta \ell$ and $\tilde{u}(t)=+1$ for $0<t<T / 2$ and $\widetilde{u}(t)=-1$ for $T / 2 \leqslant t \leqslant T$. We take $\ell_{0}=5$ and $\delta \ell=1$, and, again, $J=V_{1}=V_{2}$.

Figure 7 displays the dynamics evolution for three initial states selected as described in the main text, then evolved with the Hamiltonian of Eq. (5) of the main text but with the potential of Eq. (C1), for system size $l=12$ and $N=6$ particles. Note that, again, all three states evolve to the same stationary state. This is understood again from the flatness of the EEVs, shown in Fig. 8. Finally, a snapshot at long times of one of the states is shown in Fig. 9. Note that, despite the strong DC component of the potential, the density is spatially uniform. This is as expected, since, according to the discussion in the main text, the density at site $i$ is given by $\operatorname{tr}\left(b_{i}^{\dagger} b_{i}\right)$.

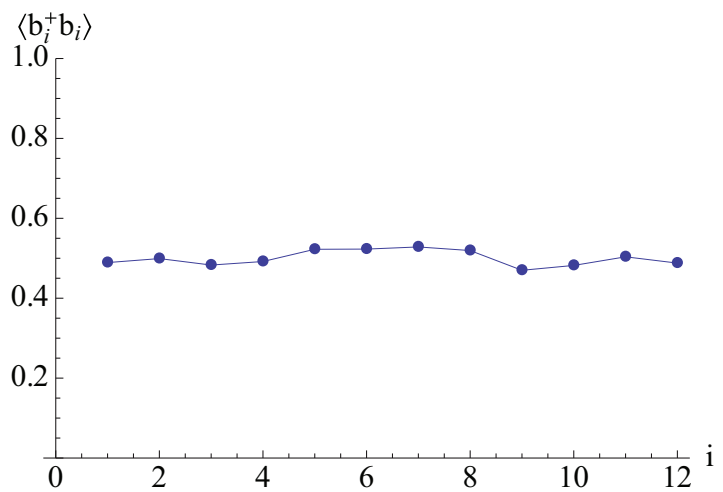

FIG. 9. (Color online) Snapshot of final density for a "breathing trap" potential, corresponding to the last point of the blue line of Fig. 7. Note the spatially uniform density despite the strong DC component in the quadratic "trapping" potential. 
[1] M. Srednicki, Phys. Rev. E 50, 888 (1994).

[2] J. M. Deutsch, Phys. Rev. A 43, 2046 (1991).

[3] M. Rigol, V. Dunjko, and M. Olshanii, Nature (London) 452, 854 (2008).

[4] S. Popescu, A. J. Short, and A. Winter, Nature Phys. 2, 754 (2006).

[5] N. Linden, S. Popescu, A. J. Short, and A. Winter, Phys. Rev. E 79, 061103 (2009).

[6] P. Reimann, Phys. Rev. Lett. 101, 190403 (2008).

[7] P. Reimann and M. Kastner, New J. Phys. 14, 043020 (2012).

[8] M. Grifoni and P. Hänggi, Phys. Rep. 304, 229 (1998).

[9] J. H. Shirley, Phys. Rev. 138, B979 (1965).

[10] H. Sambe, Phys. Rev. A 7, 2203 (1973).

[11] M. Leskes, M. Madhu, and S. Vega, Prog. Nucl. Magn. Res. Spectr. 57, 345 (2010).

[12] M. M. Maricq, Phys. Rev. B 25, 6622 (1982).

[13] S.-I. Chu, Advances in Chemical Physics, Vol. 73 (John Wiley \& Sons, New York, 1989), pp. 739-799.

[14] A. Eckardt, C. Weiss, and M. Holthaus, Phys. Rev. Lett. 95, 260404 (2005).

[15] L.-K. Lim, C. M. Smith, and A. Hemmerich, Phys. Rev. Lett. 100, 130402 (2008).
[16] A. Das, Phys. Rev. B 82, 172402 (2010).

[17] A. Russomanno, A. Silva, and G. E. Santoro, Phys. Rev. Lett. 109, 257201 (2012).

[18] A. Lazarides, A. Das, and R. Moessner, Phys. Rev. Lett 112, 150401 (2014).

[19] M. Rigol, V. Dunjko, V. Yurovsky, and M. Olshanii, Phys. Rev. Lett. 98, 050405 (2007).

[20] D. W. Hone, R. Ketzmerick, and W. Kohn, Phys. Rev. A 56, 4045 (1997).

[21] D. W. Hone, R. Ketzmerick, and W. Kohn, Phys. Rev. E 79, 051129 (2009).

[22] L. D'Alessio and M. Rigol, arXiv:1402.5141.

[23] P. Pechukas, Phys. Rev. Lett. 51, 943 (1983).

[24] W. Beugeling, R. Moessner, and M. Haque, Phys. Rev. E 89, 042112 (2014).

[25] C. Neuenhahn and F. Marquardt, Phys. Rev. E 85, 060101 (2012).

[26] We also add a very small tilt in the form of a potential $\alpha \sum_{i} n_{i}$ with $\alpha / J=10^{-3}$ to break translational invariance and remove spurious degeneracies.

[27] The averaging window is taken to be 100 states, but our results are insensitive to this width. As we shall see, the mean is independent of quasienergy for large enough systems. 